\title{
Parlementaires debout : l'interface de l'opposition politique pendant la transition en République démocratique du Congo
}

\author{
Hubert Kabungulu Ngoy-Kangoy*
}

\begin{abstract}
Résumé
Le processus de transition engagé en République démocratique du Congo en avril 1990, s'est accompagné d'une émergence et d'un foisonnement de journaux. Cette réalité a transformé les principaux coins de vente des journaux, en instance privilégiée du débat congolais, pris en otage à longueur de journée par des lecteurs infatigables communément appelés Parlementaires debout.

Cette étude essaie d'analyser le fondement théorique de ce phénomène de société où une sorte d'alliance des pauvres est très convaincue de changer le cours de l'histoire par l'appropriation de l'espace médiatique dans la lutte qui opposa singulièrement le pouvoir (Mobutu) et l'opposition (Tshisekedi) entre 1990 et 1997.

L'expérience congolaise de Parlement debout ne pourrait servir de modèle à la gestion de l'espace public africain qu'à la condition d'une « récupération intellectuelle » du phénomène susceptible de le tirer de la rue vers une réflexion plus élevée et innovante au service d'une démocratie populaire réellement participative, conclut l’étude.
\end{abstract}

\begin{abstract}
The transition process initiated in the Democratic Republic of Congo (DRC) in April 1990 was followed by the emergence and proliferation of newspapers. This reality has turned the main newsstands into a privileged Congolese debate site where indefatigable readers commonly known as "Standing-up MP's" gather all day long to comment the news. This paper attempts to analyse the theoretical foundation of the social phenomenon in which the poor, forming some kind of alliance, are strongly convinced that they can change the course of history by ap-
\end{abstract}

\footnotetext{
* Chercheur au Centre d'études politiques (CEP), Université de Kinshasa. Email : hubertkabungulu@yahoo.fr.
} 
propriating the media space in an intense struggle opposing the ruler (Mobutu) and the opposition (Tshisekedi), between 1990 and 1997.

The paper concludes that the Congolese experience of "Standing-up MP's" can serve as a model for African public space management only if it is "scholarly exploited", that is to say probably taking it off the streets for a deeper and innovative reflection at the service of a people's democracy that is truly participatory.

\section{Introduction}

Le 24 avril 1990, le Président Mobutu libère l'espace médiatique. Le débat congolais sous la transition politique qui s'installe alors en République démocratique du Congo (RDC), se cristallise autour de l'information politique entre une opposition naissante, en vue de la conquête du pouvoir et les partisans du chef de l’Etat, déterminés à conserver le pouvoir, après vingt-cinq ans de règne d'un pouvoir monolithique.

Kinshasa, la capitale de la RDC, offre encore, dix neuf ans après, à travers « les parlements debout » que sont les points de vente des journaux et leurs alentours immédiats transformés en sites de communication politique, un cadre atypique de lutte pour l'appropriation de l'espace public congolais entre le pouvoir et l'opposition. Le phénomène de « parlementaires debout » (adjectif dérivant du parlement debout), constitue à cet égard, un objet intéressant d'étude en sciences sociales, en tant qu'il fait des citoyens ordinaires, le répondant de l'opposition pendant la transition politique en vue du contrôle de l'espace public médiatique.

Habermas (2003:162) définit l'espace public comme « un espace ouvert accueillant tout discours qui s'exprime librement». Il ne s'agit pas ici de n'importe quel discours, le parlement debout est le point d'achoppement du débat congolais. D'où l'intérêt que constitue cette réalité de la sociologie politique africaine, encore mal connue et mal comprise, y compris au Congo même. Et pour cause, malgré l'importance prise par ce phénomène au fil des ans, il n'a fait l'objet que de rares réflexions scientifiques à ce jour.

Pour comprendre les facteurs explicatifs à la base de la lutte pour le contrôle de l'espace médiatique kinois (adjectif dérivant du mot Kinshasa), entre le pouvoir et ces communicateurs populaires, (les parlementaires debout), notre étude a pour objet l'analyse du fondement théorique du parlement debout au regard de son organisation ainsi que de son fonctionnement et, d'appréhender son incidence sur le débat congolais et son apport à la gestion de l'espace public africain à la lumière des évolutions successives de 1990 à 2006.

Trois méthodes, à savoir la méthode historique, la méthode systémique et la méthode analytique nous serviront d'approche. En nous plaçant dans une perspective d'avenir, la réflexion débouche sur une série de recommandations. 


\section{Historique}

\section{Concept de parlement debout}

Il semblerait que le parlement debout comme pratique plutôt que comme concept serait aussi vieux que le monde qui a vu la naissance des journaux à Kinshasa. L'avènement de la presse écrite est à situer dans la première moitié du XXè siècle (Ndaywel 1997). Mais les parlements debouts auraient connu dans l'histoire sous diverses formes, une existence lâche.

Le vocable de parlement debout désigne en RDC, particulièrement à Kinshasa, les lieux publics, notamment les coins des rues, les devantures des bâtiments publics, les abords des arrêts des bus, les proximités des sièges des partis politiques, qui servent aussi bien de lieux d'exposition quotidienne de l'information voire du journal imprimé que celui d'accès populaire à l'information politique (Kayembe 2003:8). L'épithète debout, ne sert qu'à faire la différence, par analogie, avec les parlementaires classiques, étant donné que les parlementaires debout sont dépourvus de siège, de local et discutent politique debout en plein air sous un arbre.

En effet, en créant cette instance d'autonomie, de liberté de pensée et d'expression, les Kinois avaient espéré trouver dans la rue une voie propice pour libérer la démocratie. Les médias jouèrent à cet effet un rôle éminemment politique qu'ils se transformèrent en « un lieu de prolongation du combat politique » (Kayembe 2003:204).

On peut supposer, comme dans beaucoup d'autres cas, que le vocable est d'origine médiatique. En optant pour ce mot, son initiateur avait, une idée derrière la tête, un objectif à atteindre. Albert P. (1971:74) consolide cette pensée : « La presse écrite est finalement beaucoup plus l'écho des idées et des goûts de ses lecteurs que des opinions et des choix de ses rédacteurs». Quand on envisage ce que représente un mot, on fait de la sémantique. Par contre, « en définissant un mot nous encourageons simplement les autres à l'utiliser selon nos vœux ; que le but d'une définition a rempli son rôle si elle transforme un accord sur la forme en désaccord sur le fond, donnant ainsi le champ à des nouvelles recherches » (Mills 2006:36).

Ainsi dit, en DRC, le parlement debout et par ricochet l'espace public, pourrait se définir comme le lieu de contestation, de dissidence, où se rencontrent tous ceux qui se réclamant du peuple, discutent librement des affaires de la cité. Intervenant en novembre 2008, dans l'émission Le débat africain sur radio France internationale, Mbembe A. pense que l' « espace de dissidence peut se définir comme l'acceptation de la différence dans la conception du monde, des projets de société... donc, un espace de liberté et de débat d'idées, dans la confrontation de la différence. Elle s'oppose, penset-il, à l'espace de soumission. » 
Le Parlement debout et l'adjectif qui en dérive, parlementaire debout, s'inscrivent parmi tant d'autres idiotismes qui ont alimenté le discours politique pendant la période chaude de la transition congolaise.

Selon le dictionnaire Nouveau petit Robert 1994, un idiotisme est une forme ou locution propre à une langue, impossible à traduire littéralement dans une langue de structure analogue (gallicisme, anglicisme, latinisme...). Le langage politique de la transition est très riche en la matière. Parmi les idiotismes congolais, disons le 'congolisme', nous pouvons citer quelques concepts tels que 'parti alimentaire' (parti sans assise politique), 'vagabond' ou 'vagabondage politique' (versatilité, opportunisme politique), 'perdiémiste' (profiteur politique prêt à manger à tous les râteliers), 'mouvancier' (partisan de la mouvance présidentielle), 'taupes’ (opposant et/ou partisan du pouvoir selon l'intérêt du moment), ainsi de suite.

L'histoire de la transition politique congolaise repose largement sur une base sémantique. L'intérêt des années 90 est qu'elles confèrent au parlement debout, non seulement un concept mais aussi un contenu politique réel.

\section{Evolutions successives}

Le Parlement debout résulte d'un contexte historique. Le 24 avril 1990, le Président Mobutu annonce l'ouverture démocratique suivi du multipartisme à plusieurs vitesses. Tshisekedi cristallise les mécontentements populaires face à un pouvoir déterminé à se maintenir par la force. C'est dans cette dualité que se meuvent les parlementaires debout. Les auteurs situent les origines récentes du phénomène des parlementaires debouts à des périodes différentes. Kalele les situe au lendemain de la Conférence nationale souveraine (in Nzongola et Margaret 1997:65), c'est-à-dire, plus ou moins après le 6 décembre 1992.

D’après Kalele, le premier parlement debout a vu le jour à Limete, en raison du contexte de l'après-CNS, à savoir : Limete est le lieu de résidence de Tshisekedi, symbole de résistance à la dictature mobutiste et le chef de file de l'opposition zaïroise à l'époque. Mais Limete, c'est également, le siège de l'Union pour la démocratie et le progrès social (UDPS), parti de Tshisekedi qui regroupait alors les plus radicaux des opposants (in Nzongola et Margaret 1997:68).

De l'avis de certains observateurs, le dédoublement des institutions consécutives à la nomination du gouvernement Birindwa le 2 avril 1993, peut objectivement être considéré comme la ligne droite marquant la naissance formelle du parlement debout dans sa forme actuelle. A partir de cette date, en effet, la rupture entre le pouvoir et l'opposition fut "caractérisée par l'existence dans un même pays et au même moment de : 
- deux gouvernements : le gouvernement Birindwa soutenu par le parlement du MPR (Mouvement populaire de la révolution, proche du président Mobutu) et le gouvernement Tshisekedi II élargi appuyé par le HCR (Haut-conseil de la République), parlement de transition ;

- deux parlements : le parlement du MPR attaché au chef de l'Etat et le Haut-conseil de la République acquis à la cause de l'opposition ;

- deux lois fondamentales [Constitutions] : l'Acte portant dispositions constitutionnelles relatives à la période de transition défendu par le HCR et l'Acte constitutionnel harmonisé relatif à la période de transition reconnu par le parlement du MPR » (Kabungulu 1995:148).

Au cours de cette première période de la transition, la liberté de presse contrasta avec la liberté d'opinion pendant qu'officiellement, 638 journaux étaient autorisés à paraître au cours de l'année 2000 ainsi que quelque 14 chaînes de radios et de télévision.

Les médias jouèrent un rôle éminemment politique qu'ils se transformèrent en « un lieu de prolongation de combat politique » (Kayembe 2003:204). Kalele-ka-B., de son côté, dénombre 11 parlements debout en 1996 dont huit à Kinshasa et trois dans le Congo profond. A Bukavu dans la province du Sud-Kivu, la place dite Carrefour-BBC, le rond point du carrefour Buholo IV dans la commune populaire de Kadutu, a fait tâche d'huile. Ses parlementaires ont beaucoup gêné le pouvoir. Leur dispersion par l'armée mobutiste entraîna mort d'hommes dans la première moitié des années 90.

En mai 1997, la victoire militaire de l’Armée des forces démocratiques pour la libération du Congo (AFDL) pousse Mobutu à la démission. Cette victoire est facilitée par le rejet massif du régime Mobutu par les Congolais. Tshisekedi et l'UDPS se lèvent alors contre ce qu'ils ont appelé « la dictature de Kabila » (Kabungulu 2006:132). Le pouvoir n’hésite pas à recourir à des méthodes fortes. L'opposition se terre. Le parlement sans véritable repère, à défaut de s'émanciper, se fait l'écho de Tshisekedi qui par moment essaie de relever la tête.

Le retour en force des tutsi par le truchement l'Alliance des forces démocratiques pour la libération du Congo en 1997, suivi de la limitation à quatre des partis politiques à partir de fin janvier 1999 irritent les intellectuels et les partis politiques en général. Les parlementaires debout s'érigent alors en opposition parfois ouverte contre le régime. Les Comités du pouvoir populaire (CPP) crées par le nouveau régime en avril sont tournés en dérision par ces les parlementaires debout.

Rappelons qu’à Kisangani, les parlementaires debout s'érigèrent en une véritable opposition à la « rébellion » ouverte le 2 août 1988 contre le régime de Kabila père. Il s'en suivit, l'interdiction d'attroupements, des actes de 
répression. Le pouvoir de Kabila exerça toutes sortes de pression sur les parlementaires debout. A la suite de sa mort, son successeur, Joseph Kabila relance le débat politique après l'hibernation des partis entre 1997 et 2001 (Kabungulu 2006:122-140).

En 2003, un sociogramme des sites de parlements debouts établit par le journal Liberté à travers la ville de Kinshasa, en a dénombré 15 : Quartier Mikondo, commune de N'Sele ; Zando ya Hindu, Arrêt Masina/Kingasani ya Suka ; Arrêt Masina/Kingasani Pascal sur le Boulevard Lumumba ; Quartier 1, commune de N'Djili (Eucalyptus) ; Quartier N’Djili Sainte Thérèse ; Quartier Mongo, commune de Matete ; Quartier Super-Lemba ; Quartier LembaEchangeur ; 12ème rue Limete, Boulevard Lumumba ; Environnement immédiat de la résidence de Tshisekedi, $10^{\text {ème }}$ rue Limete ; Matonge, commune de Kalamu au croisement des avenues Kasa Vubu ; Wenze ya Bayaka, commune de Ngiri Ngiri ; Bandal Moulaert et Kintambo (Kayembe 2003:290).

Examinons la nature du phénomène.

\section{Caractéristiques}

\section{Lieu de contestation}

Il convient de noter que le parlement debout se veut le lieu de communication politique. C'est l'événement politique qui détermine la nature du débat. N'Djili Eucalyptus a été identifié comme le parlement debout le plus actif et le plus extrémiste de la capitale, qui, n'avait d'égal à l'intérieur du pays que « BBCCarrefour » à Bukavu. A Kinshasa, Lemba-Echangeur, face à la station service Elf, appelé « Place le laboureur et ses enfants » a toujours été cité parmi les sites les plus redoutables.

\section{Endroit porteur des messages forts}

Il y a lieu de faire observer que la plupart des parlements debout se situent à « des endroits porteurs des messages significatifs » (Kayembe 2003:290) : la proximité d'un arrêt de bus, les terminus des transports en commun ou des points relais, la proximité des certains sièges de partis politiques, des bâtiments publics, des quartiers chauds et l'emplacement le long des artères de grand trafic et à l'entrée des 'cités paradis' (Kayembe 2003:292) ou populaires.

Le message fort est par essence un message captivant, fort, susceptible d'attirer l'attention. A travers La fabrication de l'information, Aubenas F. et Benasayag M. (1999:18), stigmatisent le fait que l'objectif d'un tel message n'a pour but que de « réveiller la population et 'provoquer l'effet d'un signal d'alarme'».

On a également assisté à la délocalisation et surtout à la scission de certains parlements debouts. 


\section{Scissiparité}

Les parlements debout ont parfois été caractérisés par des désaccords d'option politique jusqu'à leur scission. La séparation était consécutive fondamentalement à la crise de confiance et de la méfiance réciproque. Le parlement de la $12^{\mathrm{e}}$ rue a ainsi donné naissance à celui de la $10^{\mathrm{e}}$ rue Limete, suite à la dislocation entre Tshisekedi (opposition radicale) et son ancien compagnon de lutte, Kibassa Maliba (devenu opposition modéré), allié au pouvoir de Mobutu dans la première moitié des années 90 . Après les divisions et les contradictions internes au sein de l'UDPS, les parlementaires debout ont commencé à évoluer presque sans référence à une quelconque ligne de conduite.

\section{Organisation et fonctionnement}

\section{Statut et rôle}

Dans sa forme originelle, le parlement debout fut organisé de façon plus ou moins cohérente. Les parlementaires debout étaient constitués de toutes les catégories des couches de citoyens. On y retrouvait diplômés, analphabètes, civils, militaires, travailleurs, retraités, professeurs d'universités désargentés.

Au niveau d'ensemble, d'après Kalele, le parlement debout était supervisé par un coordonnateur en chef. Il se réunissait à Limete une fois par semaine avec les membres de ses sept états-majors, le jeudi en assemblée générale, après le conseil du "gouvernement légal » parallèle dirigé par Tshisekedi. Dans la distribution des rôles, chaque parlement était constitué d'un président, d'un secrétaire-rapporteur, des messagers spéciaux et des membres. Le président jouait le rôle de modérateur par la distribution de la parole, commentait l'information et proposait des actions concrètes à mener. Les messagers spéciaux étaient chargés de transmission des informations urgentes par système de relais. Les autres membres avaient le devoir de répercuter chaque information apprise au parlement, dans leurs quartiers respectifs. La lecture se faisait à haute voix par un membre à l'intention de tout le groupe ; suivie des commentaires en lingala (la langue courante parlée à Kinshasa), pour permettre à tout le monde de bien comprendre et de s'exprimer (in Nzongola et Margaret 1997:67).

A l'analyse, comme organisation fonctionnelle (l'opposée d'organisation hiérarchique), la structure du parlement debout conférait à ses membres un nouveau rôle et statut dans la société congolaise en crise. D’après Grawitz (2002:359), le concept de rôle est « Introduit [en psychologie sociale] par Binet en 1898, repris par F. Mead, Linton puis Moreno. Par analogie avec l'acteur, au théâtre : ensembles de comportements relatifs à une position, fixés par la société (rôle du père, du maître) et que l'on s'attend à voir jouer 
(expectation), par ceux qui la détiennent ». En fait, « Le rôle définit ainsi une zone d'obligations et de contraintes corrélative d'une zone d'autonomie conditionnelle » (Boudon et Bourricaud 1982:505).

En s'accaparant de l'information politique, les parlementaires debout jouaient, dans une certaine mesure, surtout pendant la période forte de la transition, le rôle d'éclaireur, de porte-voix, de voix des sans voix. Devenus fidèles alliés de l'opinion populaire, pour paraphraser Kapferer J.H., privés de vie publique, la rue rendait publique la vie des parlementaires debout (Kapferer 1998:177). Quant à leur statut, il peut se concevoir comme celui des redresseurs de torts, d'auxiliaires de l'opposition radicale, de garantie ultime, voire l'expression de la volonté populaire. Le statut et le rôle du parlementaire debout, lui confèrent ainsi une sorte de contrat social tacite avec la population.

Figure 1 : Contrat social entre population et parlementaires debout

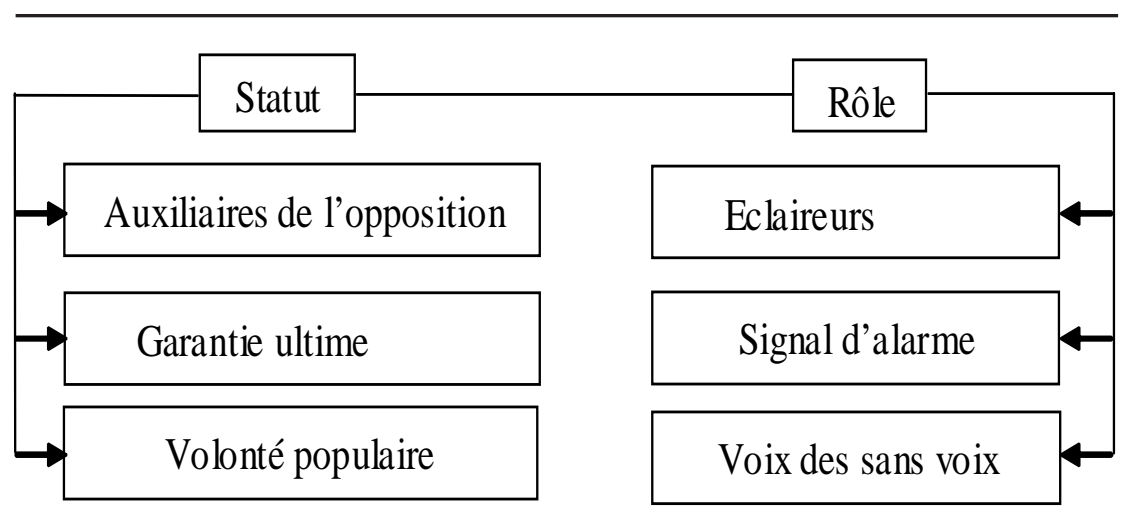

Le status, « est utilisé en psychologie sociale en place de statut pour indiquer la situation de l'individu, son rang dans une société donnée, le rôle qui l'incombe, la fonction sociale qui lui est dévolue » (Grawitz 2002:382) que l'auteur qualifie d'aspect fonctionnel et normatif. La ligne de séparation n'est pas toujours facile à établir entre le statut et le rôle exercés par un individu ou par un groupe d'individus dans la société. Ainsi donc, «L'expression de statut désigne la position qu'un individu occupe dans un groupe, ou qu'un groupe occupe dans une société (entendu comme groupe de groupes)...On peut combiner ces deux indications en définissant le statut comme l'ensemble des relations égalitaires et hiérarchiques qu'un individu entretient avec les autres membres de son groupe (Boudon et Bourricaud 1982:564), en vue d'un objectif à atteindre. 


\section{Stratégie d'action}

Les parlements opéraient selon une stratégie d'action et une règle de conduite précises. Les journaux en tant que source ouverte, constituent la matière première brute des parlementaires debout. A cet effet, ils ont été, du moins aux origines, les porte-voix de l'opposition incarnée par l'UDPS, mais ils n'étaient tendres ni envers le pouvoir ni envers l'opposition. Cependant, dans la scène politique congolaise, particulièrement des années 90 , véritable panier à crabes, les parlementaires debout eux-mêmes étaient parfois fourvoyés dans leurs analyses « devant trois types d'acteurs politiques :

- les «Mouvanciers » (proches du Chef l'Etat),

- les « Opposants » (théoriquement il y a plusieurs Oppositions),

- les « Taupes » (faux Opposants ou faux Mouvanciers) (Kabungulu 1995:265).

De tous les temps, "Au Zaïre où la politique constitue un moyen d'enrichissement, de survie, et même pour beaucoup, une profession... La corrélation entre les facteurs objectifs et subjectifs complique tout effort d'analyse » (Kabungulu 1995:265), puisqu'on peut se réveiller le matin opposant pour devenir inconditionnel allié du pouvoir le soir.

Voilà pourquoi à Kinshasa, la rue devint une sorte de réponse à la faillite de l'Etat (de Villers et Omasombo 1997:223, 245) et de l'opposition. On a pu observer, dans ce cas, que, l'agir communicationnel pouvait se traduire par trois types d'attitudes possibles :

- accord parfait rue-opposition : plus la cause était juste, plus l'unité au sein de la population était acquise. L’action de la rue se solde par un succès ;

- désaccord total rue-opposition : la population désapprouve le point de vue de l'opposition, la cause est perdue d'avance ;

- divergences rue-opposition : la population est divisée sur une opinion soutenue par l'opposition, le résultat est mitigé.

Est-il qu'à l'origine, l'action des parlementaires debout avait comme idéeforce, le discours politique de Tshisekedi. Ce discours pouvait se traduire par la formule suivante : « Mobutu = le mal zaïrois et la cause de toutes les misères du peuple. Tshisekedi = le libérateur du peuple et la fin de la misère. Sa stratégie : chasser Mobutu du pouvoir " (N’Gbanda 1995:134). Mais cette stratégie n’a pas toujours été payante, de sorte que « Tshisekedi a été en fait à la fois prisonnier et victime de sa propre stratégie et de son discours politique » (N’Gbanda 1995:134). Accusé de corruption ou de complicité, il en paya le frais toutes les fois qu'il se montra conciliant vis-à-vis du pouvoir. Ce sont les sept états-majors qui, ensemble, imaginaient des stratégies à mener. 
Dans la pratique, toute l'action des parlementaires reposait sur une espèce de propagande et de manipulation manichéenne, par la diabolisation du pouvoir (incarnation du mal) contre la sacralisation de l'opposition (incarnation du bien), bref une lutte entre la force du bien et la force du mal.

Figure 2: Technique de propagande des parlementaires debout

\begin{tabular}{ll}
\hline Pouvoir & Opposition \\
Force du Mal & Symbole du Bien \\
(Diabolisation) & (Sacralisation) \\
\hline
\end{tabular}

Hazan (2006:91) considère comme « Les véritables opérations de manipulation de l'information, celles que l'on qualifie de 'désinformation', s'appuient sur des informations véritables pour en créer ou en justifier d'autres, farfelues ». Par contre la propagande, pour Le journal du dimanche du 5 décembre 2004, c'est cette stratégie consistant à présenter l'opposition « comme une sorte de bon papa collectif, sévère mais bienveillant, fermement décidé à faire régner la justice pour le bonheur des populations » (Hazan 2004:75).

Inattendument, dans cette lutte, tout dérapage présumé ou supposé face à l'idéal démocratique, d'où qu'il vienne, devait faire l'objet de rappel à l'ordre. Ce qui conduisit à des multiples abus et à l'intolérance politique envers le pouvoir ou envers l'opposition.

Finalement, la question qui se pose face à ce phénomène est celle de savoir, pourquoi des responsables de famille passent-ils l'essentiel de leur temps à discuter politique à longueur de journée. Sur le plan théorique, cette réalité ne pouvait s'expliquer que par rapport à la situation politique et socioculturel du citoyen congolais.

\section{Fondement théorique du 'parlementarisme debout'}

\section{Du point de vue politique : une lutte idéologique}

Les Congolais ayant longtemps souffert du monolithisme et de l'unanimisme, ont vite compris à l'aube de 1990 que, celui qui détient l'information détient le pouvoir. En fait, « La politique est par définition, communication (Virieu 1990:50). Le 'parlementarisme debout' se veut un mode particulier de participation politique. Ce mode de participation relèverait d'une lutte idéologique, pour l'appropriation de l'espace public par le contrôle de l'information politique. Selon Capdevila (2004:25), en effet, « Il est impossible qu'une prise de conscience s'effectue autrement qu’à travers un code 
idéologique. Ainsi l'idéologie est-elle affectée par la schématisation inéluctable qui s'attache à elle ; en s'attachant elle-même se sédimente, alors que faits et situations changent ».

Il s'agit d'une forme de participation consciente et /ou inconsciente, une forme d'auto-prise en charge par la démocratie participative. D’où, toutes sortes de contradictions et conflits d'intérêts avec le pouvoir aussi bien de Mobutu, de Kabila père que de Kabila fils. Il est évident que « Le mentale fabrique l'idéologie, l’idéologie domestique les esprits » (Ramonet 2004:10). Et comme légitimation, il va de soi, qu'en intégrant dans ses raisons d'agir le comportement et les motivations des autres, «l'idéologie est inséparable de conflits d'intérêts qui minent le consensus social » (Capdevila 2004:25).

Comme le dit Edmond Jouve, les problèmes politiques sont les problèmes de tout le monde et les problèmes de tout le monde sont des problèmes politiques. En discutant politique, les parlementaires debout entendaient imposer leur point de vue dans le débat congolais, plutôt qu'à s'engager dans la lutte du pouvoir, moins encore à participer à sa gestion. Ils ont toujours perçu la politique comme l'art de mentir.

\section{Du point de vue social : une lutte des classes}

La démocratie pour les Congolais en général et pour les parlementaires debout en particulier était synonyme de mieux-être, susceptible de permettre l'accès au manger, aux soins de santé et à l'éducation pour tous. Par conséquent, toute obstruction à la démocratie était synonyme de perpétuation de la misère du peuple. Or, Mobutu était considéré comme le principal goulot d'étranglement à cet avènement. En tant que vision futuriste, le parlement debout participe de la lutte quotidienne kinoise pour l'indépendance du ventre, du lingala, 'kobeta libanga' (casser la pierre). Par néologisme, cela s'appelle, le 'libanguisme' (la lutte pour la survie).

Le parlement debout apparaît donc comme un processus de socialisation, une alliance des pauvres à la recherche d'un lendemain meilleur. La socialisation étant entendue comme « le processus d'assimilation des individus aux groupes sociaux (Boudon et Bourricaud 1982:527). Par le fait de développer des relations sociales, de s'adapter et de s'intégrer à la vie du groupe, le parlement debout pouvait s'interpréter comme un moyen de lutte pour la survie.

C'est ainsi que, par instinct de protection contre l'ordre ancien, dans une ville de Kinshasa habitée par une majorité de laissés pour compte, le 'parlementarisme debout' ne recruta que parmi la couche sociale pauvre. Il est évident qu'au-delà de facteur d'intégration, dans une société donnée, la socialisation « représente un apprentissage et un ajustement ", écrit Grawitz M. (2004:374), face aux valeurs, aux normes et aux codes symboliques de ladite société. 


\section{Du point de vue culturel : une quête identitaire}

Dans leur idéalisme, en faisant entendre leur voie, les parlementaires debout croyaient de la sorte, pouvoir affirmer leur identité par rapport à une société matérialiste, minée par des antivaleurs ainsi que par la dépravation des mœurs politiques et sociales.

L'identité est définie par Warnier (2004:23) comme «l'ensemble des répertoires d'action, de langue et de culture qui permettent à une personne de reconnaître son appartenance à une certain groupe social et à s'identifier à lui ». D'où, le contrôle de l'information devient le principal point d'achoppement entre le pouvoir et le parlement debout. Il est évident, indique l'auteur, que « les questions posées par des conflits politiques émergent dans le débat politique sous forme des revendications politiques et que dans les médias, la politique apparaît comme un ressort important de ces revendications identitaires » (Warnier 2004:99-100).

Par revendications identitaires, il faudrait entendre d'après Warnier J.-P. (2004:99-100), « les conduites identificatrices revendiquées par un groupe de manière agressive au regard des autres groupes, et autoritairement conformistes à l'intérieur. Ces réactions, sont une réponse suscitée par la menace de l'autre sur les cultures singulières des groupes ».

Ce qui est en cause ici, c'est la domestication groupale de l'information par les parlementaires debout, que Bianchi J. et Bourgeois H. (1992) identifient traditionnellement à l' ' appropriation du public » (in Kayembe 2003:9). Les auteurs assimilent les dynamiques d'appropriation, disons l'usage que les parlementaires debout font de l'information, comme « une donnée de la situation culturelle en face de laquelle le public se prononce et réagit...cherche à montrer ce qui se passe dès qu'un écran s'allume, qu'un écouteur fonctionne ou qu'une page imprimée se donne à lire » (Kayembe 2003:9).

Et avec Dereze G. (1997), on s'aperçoit que toute pratique culturelle renvoie à un « assemblage plus ou moins cohérent, fluide, d'éléments concrets ou idéologiques (religieux, politiques), à la fois livrés par une tradition (celle de famille, de groupe social) et réactualisés au jour le jour à travers les comportements traduisant dans une visibilité sociale des fragments de ce dispositif culturel ; pratique est la capacité de ce qui est décisif pour l'identité d'un usage ou d'un groupe pour autant que cette identité lui permette de prendre place dans le réseau des relations sociales inscrites dans l'environnement » (in Kayembe 2003:195). Le parlement debout équivaut, ici, à une forme d'affirmation de soi.

Mais, qu'est-ce qui pourrait expliquer la survivance d'un tel phénomène ? 


\section{Mécanisme de reproduction}

La reproduction du phénomène de parlementaires debout, semble avoir obéi à une double logique de dynamique de groupe (ou dynamique des groupes), entendu sociologiquement, comme un ensemble des règles qui président à la conduite des groupes sociaux dans le cadre de leur propre activité. En clair, face aux motivations et aux influences, il s'agit de « forces de cohésion qui maintiennent les individus dans le groupe, formes de l'autorité qui conduisent le groupe à réaliser plus ou moins bien ses tâches, influences qui s'exercent sur les individus lors de leur passage par le groupe » (Stoetzel 1971:200).

Dans leur Dictionnaire critique de la sociologie, Boudon et Bourricaud (1982:504) font observer que « Les phénomènes de reproduction - c'est-àdire d'équilibre - sont aussi difficiles à expliquer que les phénomènes de changement et de déséquilibre. Ils comportent une double tentation : celle de l'explication téléologique, et du recours à l'analogie organiciste ».

Ainsi, distinguons-nous comme à la base de reproduction du phénomène étudié :

- Les besoins de renouvellement : ils résulteraient des interactions et contradictions internes entre parlements ou parlementaires, face à l'ensemble des règles qui président à la conduite du groupe (égalité, respect mutuel, tolérance, camaraderie, fraternité, nationalisme,...) dans la mesure où « les groupes possèdent leur culture propre, comportant des différentiations préétablies, des normes et des valeurs, des rites, mais encore qu'ils se raccordent à une société plus vaste, et qu'ils s'intègrent dans des rouages plus généraux » (Stoetzel 1971:204). La faiblesse des parlements debout tiendrait à leur nature, la fonctionnalité (sur la base affective) de sympathie, d'antipathie ou d'indifférence, comme petits groupes artificiellement constitués au lieu d'une hiérarchisation (sur la base d'autorité) suivant une ligne de conduite fondée sur la discipline et la sanction par exemple.

- Les besoins de communication : ils relèveraient de l'impératif de sensibilisation par le contrôle de l'information politique mais aussi, en vue d'établir entre parlements des échanges d'informations ou des communications. Le parlement se réduit en l'espèce, à une instance de transmission de pensée ou de ce qui est considéré, à tort ou à raison, comme la volonté du peuple. La survivance du parlement debout, s'apparente alors, à une réponse d'inadaptation des réponses politiques aux exigences de la démocratie. Il est donc établi que «l'activité de communication politique issue de l'enracinement du parlement debout dans l'espace public est la manifestation d'une insatisfaction d'une part et la lutte contre la 'politisation' du système général de l'information politique (variable exogène) à partir d'une politisation des espaces publics (variable endogène) d'autre part (Kayembe 2003:489). 
En dialectique, la dynamique renvoie à un ensemble des forces en interaction et en opposition dans un phénomène, une structure. Stoetzel J. (1971:203) l'affirme sans équivoque : « Le système des communications est donc en relation, à la fois comme cause et comme effet, avec un autre aspect de la structure, celui des statuts et des rôles » des parlementaires debout. La tendance est grande d'attribuer le phénomène du parlement debout à l'exceptionnalisme ou au particularisme congolais. Repris par Kayembe, (2003:195), Ekambo J. fait remarquer à cet égard, qu' «il ne peut être pas complètement établi que la problématique de la groupalité dans l'usage de l'information soit opératoire exclusive à la société congolaise ou africaine » (www.u-grenoble 3.fr, avril 2004).

En effet, devant cette double nécessité commandée par l'évolution du processus de transition, on a assisté à l'émergence de plusieurs types des détenteurs du pouvoir de la rue, selon une autre expression consacrée au Congo.

\section{Typologie de parlementaires debout}

La société congolaise sous la transition, est une société en perpétuelle mutation, caractérisée par des évolutions politiques rapides dont l'année 1990, comme nous l'avions dit, constitue la charnière, partant de Mobutu (multipartisme à trois au multipartisme intégral) à Laurent-Désiré Kabila (hibernation politique, retour au monopartisme puis au multipartisme à quatre) et à Joseph Kabila (un multipartisme limitatif, puis le multipartisme intégral). Chez Comte et Spencer, statique sociale (équilibre des sociétés) s'oppose à la dynamique sociale (progrès des sociétés) (Lalande 1985:255), cette dernière étant la partie de la sociologie qui étudie les faits en évolution et non dans leur état actuel.

Or, on peut à juste titre considérer les parlementaires debout comme le baromètre de la transition. En s'appuyant sur la dynamique sociale en tant qu'elle traite du progrès des sociétés (Grawitz 2004:135), nous pouvons distinguer trois types de parlementaires debout.

- Le type fanatique : il se distingue par une attitude militante parfois surréaliste. Cette typologie comprendrait les parlementaires debout de la première génération des années 90-97. Tshisekedistes jusqu'à la moelle des os, ils firent preuve d'intransigeance, de nature irréconciliable et très fanatique, prêt à tout. Le type fanatique eut plusieurs échauffourées avec l'armée ou la gendarmerie à la 12è rue Limete. BBC-Carrefour à Bukavu a beaucoup gêné le pouvoir. La dispersion des parlementaires debout par l'armée mobutiste au temps fort de la transition y entraînera mort d'hommes.

Mobutu, alors en baisse de popularité jamais connue parmi les Congolais, les parlementaires debout jouissent d'une double confiance de la part de la population : en tant que le porte-parole de l'opposition radicale et en sa qualité de relais des journaux. La majorité de la population constituée d'analphabètes, a toujours assimilé l'opinion des médias à une parole d'évangile. 
- Le type attentiste : en dépit de la demande politique, son comportement a été théoriquement marqué par une certaine attitude prudente. Dubitatif, il vit dans l'expectative, accordant au pouvoir le bénéfice du doute au regard de son intention de mener jusqu'au bout le processus démocratique. S'il se réserve souvent de l'action de rue, c'est par peur de la gâchette facile de l'armée de l'AFDL et non par allégeance au nouveau régime décrié, par les deux ténors de l'opposition, Tshisekedi (UDPS) et Gizenga, du Parti lumumbiste unifié (PALU), qui lui reprochèrent le retour à la dictature.

Son attitude est marquée par une certaine réserve face aux rapports de forces qui l'oppose au pouvoir, particulièrement de Kabila père (1997 à 2001). Mais ceci n'a pas empêché aux parlementaires kinois de s’insurger contre son régime accusé de connivence avec les Tutsi ou contre le RCD (Rassemblement congolais pour la démocratie) en 1998, malgré l’usage abusif de la force. Dans les deux cas, on assista à l'interdiction d'attroupements, la répression des parlementaires afin de les décourager dans leurs initiatives.

En tous temps et en toutes circonstances, à l'instar d'un cadavre de vipère, mort ou vivant, chaud ou froid, le parlementaire debout fit toujours peur, dérange.

- Le type associatif et communicatif : cette troisième génération des parlementaires debout (2001-2006) se distingue, par une attitude expansive. C'est un prototype qui aime à communiquer ses sentiments. Il est expansif et proche de l'esprit associatif, à la limite, se complait-il à la résignation. Le parlement debout se réduit de plus en plus à un club d'amis. En plus de l'actualité politique, il s'y dispute toutes sortes de commérages : musique, football, faits divers, etc. Le parlement debout offre ainsi un cadre où les sans emplois peuvent se retrouver pour perdre le temps, 'boma l'heure', selon l'expression consacrée en langue lingala.

Que retenir de tout ce qui précède ?

\section{Conclusion}

Beaucoup d'éléments de réponses peuvent être tirés de l'analyse par rapport à notre sujet : Parlementaires debout : l'interface de l'opposition politique pendant la transition en République démocratique du Congo.

- Primo : Les parlementaires debout sont une force inclusive de la transition et un dérivatif à la crise de confiance entre le pouvoir et le peuple.

- Secundo : Instance de dissidence, initialement conçu comme espace populaire de communication pour libérer la démocratie (1990 à 1997), le parlement debout a joué le rôle de contre-pouvoir et d'auxiliaire d'opposition. Même s'il subsiste au fil de l'évolution de la transition, après la chute du régime Mobutu en 1997 et les élections démocratiques de 2006, le parlement debout s'émousse et se fragilise, quand bien même il inquiète toujours le pouvoir. 
- Tertio : En ce qui concerne son contenu intellectuel ou le fondement théorique du parlement debout, nous venons de démontrer, sur le plan politique que le phénomène devrait être compris comme une lutte idéologique, un mode de participation politique pour le contrôle de l'espace médiatique. Sur le plan social, il est une lutte d'hommes, qui, à travers un processus particulier de socialisation, luttent contre l'injustice sociale. Sur le plan culturel, le phénomène se réduirait à la recherche d'une nouvelle identité du groupe, dans une société qu'il considère comme sans repère.

- Quarto : Effectivement, quant à l'impact du parlement debout sur l'avènement de la démocratie, il est à la fois relatif et subjectif. D'une part, il n'est pas quantifiable. D'autre part, le degré de conviction des parlementaires eux-mêmes a été pour le moins variable, selon qu'on est fanatique, attentiste ou communicatif. Là aussi, la ligne de démarcation est loin d'être tranchée, qu'elle se complique par l'existence des modérés et des extrémistes dans un camp comme dans un autre. En dépit de son rôle symbolique, le moins que l'on puisse dire, est que le parlementaire s'est efficacement assumé en tant que système d'alarme fort contre tout dérapage ou obstacle sur le chemin de la démocratisation. En revanche, les parlements debout se sont indistinctement illustrés par une lutte au niveau verbal où l'influence de la psychanalyse s'est avérée plus ou moins forte. Une chose est sûre, la vocation des parlementaires debout a été proportionnelle au déficit démocratique.

- Quinto : De par sa spécificité, bien que limitée, le parlement debout apporte une réelle contribution au débat africain. Il est à la fois un cadre de liberté, de pensée et d'expression de la confrontation, entre les intelligences de l'intelligence politique publique et les intelligences de l'intelligence politique privée. A cet égard, il a pu être observé un déficit du leadership pendant la longue transition congolaise, déficit révélateur d'une crise de confiance entre la population et ses dirigeants, toutes tendances confondues.

Toutes proportions gardées, en guise de conclusion, l'expérience congolaise du parlement debout ne pourrait servir de modèle à l'espace public africain qu'à certaines conditions ci-après :

- la récupération intellectuelle du parlement debout par l'élite consciente, en vue d'en faire le lieu de réflexion constructive sur la gestion quotidienne de la cité ;

- le développement de la réflexion sur le phénomène du parlement debout à travers des échanges et discussions entre différents centres de recherche pour une approche africaine innovante de la démocratie participative ; 
- le rappel à l'ordre de l'agir politique des dirigeants africains, par l'agir communicationnel à travers des rencontres et regroupements régionaux des parlements debout, en vue de la quête des solutions concertées, susceptibles d'élever le débat et de créer une synergie face aux discours officiels.

Devons-nous parler d'un rêve ou d'une utopie ? Peut-être, mais au commencement était la parole et la parole se fit chair.

\section{Références}

Albert, P., 1971, La presse, Paris, Presses Universitaires de France.

Aubenas F. et Benasayag, M., 1999, La fabrication de l'information. Les journalistes et l'idéologie de la communication, Paris, La Découverte.

Barzun, J. and Graff, H., 1957, The Modern Research, New York, Harcourt Brace.

Bianchi, J. et Bourgeois, H., 1992, Les médias côté public. Le jeu de la réception, Paris, Centurion.

Bourdon, R. et Bourricaud, F., 1982, Dictionnaire critique de la sociologie, Paris, Presses universitaires de France.

Capdevila, N., 2004, Le concept de l'idéologie, Paris, Presses Universitaires de France.

Dereze, G., 1997, « De la vie sociale des récits médiatiques. Une ethnosociologie focalisée », Recherche en communication, No 7.

De Villers G. et Omasombo, T.J., 1997, « Quand le peuple kinois envahit la rue... », in Trefon, T., éd., Ordre et désordre à Kinshasa. Réponses populaires à la faillite de l’Etat, Cahiers Africains, No. 61-62, pp. 223-245.

Ekambo, J.-C., « La problématique des anciennes technologies de la communication (ATC) africaines dans l'espace communicationnel contemporain » in Enjeux 3.fr avril 2004, p.4, consulté le 12 octobre 2002.

Grawitz, M., 2002, Lexique des sciences sociales, 8è éd., Paris, Dalloz.

Habermas, J., 1992, «L'espace public 30 ans après », in Quaderni. La Revue de la communication, No 18, p. 162.

Hazan, E., 2006, La propagande du quotidien, Paris, Raison d'agir.

Kabungulu, N.-K., 1995, La transition démocratique au Zaïre, avril1990-juillet 1994, Kinshasa, CIEDOS Université de Kinshasa.

Kabungulu, N.-K., 2006, « Parties and Political Transition in The Democratic Republic of Congo », EISA research Report, No. 20, pp. 16-34.

Kalele-ka-B., 1997, « La démocratie à la base. L'expérience des parlementairesdebout », in Nzongola, G. et Margaret, C., ed., The State Democraty in Africa, Harare, AAPS Books, pp. 64-73.

Kapferer, J.-N., 1987, Les Rumeurs. Le plus vieux média du monde, Paris,Seuil.

Kayembe, A., 2003, Les dynamiques d'appropriation de la communication politique sur les places publiques à Kinshasa, Thèse de doctorat, Kinshasa, Facultés catholiques de Kinshasa, ronéo. 
Lalande, A., 1985, Vocabulaire technique et critique de la philosophie, Paris, Presses universitaires de France.

Le journal du dimanche, 5 décembre 2004.

Mills, W.C., 2006, L’imagination sociologique, Paris, La Découverte/Poche.

Ndaywel, I., 1997, Histoire du Zaïre. De l'héritage ancien à l'âge contemporain, Bruxelles, Duculot.

N’Gbanda, H., 1995, La transition au Zaïre. Le long du tunnel, Kinshasa, NORAF.

Pronovost, G., 1996, Médias et pratiques culturelles, Paris, Presses universitaires de Grenoble.

Ramonet, I., 2004, Propagandes silencieuses, Paris, Gallimard.

Stoetzel, J., 1971, La psychologie sociale, Paris, Flammarion.

Virieu, F.-H., 1990, La médiacratie, Paris, Flammarion.

Warnier, J.-P., 2004, La mondialisation de la culture, 3è édition, Paris, La découverte. 